\title{
THEORY OF EDGE RADIATION. PART I: FOUNDATIONS AND BASIC APPLICATIONS
}

\author{
G. Geloni, V. Kocharyan, E. Saldin, E. Schneidmiller and M. Yurkov, DESY, Hamburg, Germany
}

\begin{abstract}
We formulate a complete theory of Edge Radiation based on a novel method relying on Fourier Optics techniques. Special attention is paid in discussing the validity of approximations upon which the theory is built. Our study makes consistent use of both similarity techniques and comparisons with numerical results from simulation. We discuss both near and far zone. Physical understanding of many asymptotes is discussed. As an example of application we discuss the case of Transition Undulator Radiation, which can be conveniently treated with our formalism. This work forms the theoretical basis for understanding the impact of Edge radiation on XFEL setups, which is discussed in another contribution to this conference. These proceedings are based on the article [1], to which we address the interested reader for further information and references.
\end{abstract}

\section{INTRODUCTION}

Synchrotron Radiation (SR) sources from bending magnets are brilliant, and cover the continuous spectral range from microwaves to X-rays. However, in order to optimally meet the needs of basic research with SR, it is desirable to provide specific radiation characteristics, which cannot be obtained from bending magnets, but require insertion devices. These are installed along the particle beam path between two bending magnets, and introduce no net beam deflection. Therefore, they can be incorporated in a given beamline without changing its geometry. Undulators are a typical example of such devices, generating specific radiation characteristics in the short wavelength range.

The history of SR utilization in the long wavelength region (from micrometer to millimeter) is more recent than that in the short wavelength range. Long wavelength SR sources may have a strong potential for infrared spectroscopy or imaging techniques. In fact, they are some order of magnitude brighter than standard thermal sources in the same spectral range.

Large angles are required to extract long wavelength SR from bending magnets, because the "natural" opening angle in this case increases up to several tens milliradians in the far-infrared range. However, the situation changes dramatically if a straight section is introduced between two bends, like in Fig. 1(a). Long-wavelength radiation emitted by relativistic electrons in this setup is called Edge Radiation (ER), and presents a significantly smaller opening angle than standard SR from bends.

$\mathrm{ER}$ and bending magnet radiation have equivalent flux and brightness. In fact, the physical process of ER emis- sion is not different from that of radiation emission from a single bend. However, radiation from the setup in Fig. 1(a) exhibits special features, due to a narrower opening angle of ER over SR from bends. Although for many experiments using infrared radiation one can accept large collection angles in the horizontal and vertical directions, as the wavelength gets longer ER can be advantageous in terms of simplicity of the photon beamline e.g. in infrared microspectroscopy applications.

\section{FOUNDATIONS}

ER theory is a part of the more general SR theory, very much like Undulator Radiation (UR) theory is a part of SR theory. Similarly to the UR case, also for ER the knowledge of the applicability region of the far-field formulas and corrections for near-field effects are of practical importance. In most practical cases, the distance between ER source and observer (i.e. the first optical element of the photon beamline) are comparable or even much smaller than the length of the straight section, which plays the role of the length of the insertion device for ER. In [1] we developed a theory of near-field ER based on Fourier Optics (FO) techniques. These techniques can be exploited without limitations for ER setups, because the paraxial approximation can always be applied in the case of electrons in ultra-relativistic motion. The use of the paraxial approximation allows reconstruction of the field in the near-zone from the knowledge of the far-field data. The solvability of the inverse problem for the field allows characterization of any ER setup, starting from the far-zone field, in terms of virtual sources. These sources exhibit a plane wavefront, and can be pictured as waists of laser-like beams. Using this kind of description we develop our theory in close relation with laser-beam optics. In particular, usual FO can be exploited to characterize the field at any distance, providing a tool for designing and analyzing ER setups. This program was carried out in detail in [1].

\section{Similarity Techniques}

ER theory can be introduced intuitively and simply by simulating the spectral energy density per unit angle as a function of observation angles for the geometry in 1(a), and studying the parameter space of the problem. For this purpose we take advantage of the code SRW by Oleg Chubar, which provides a numerical solution of Maxwell's equations.

The origin of a Cartesian coordinate system is placed at the center of straight section. The $z$-axis is in the direction of straight section. Parameters of the problem are the 
(a)

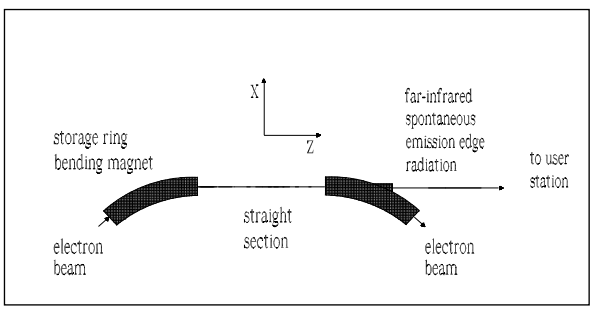

(b)

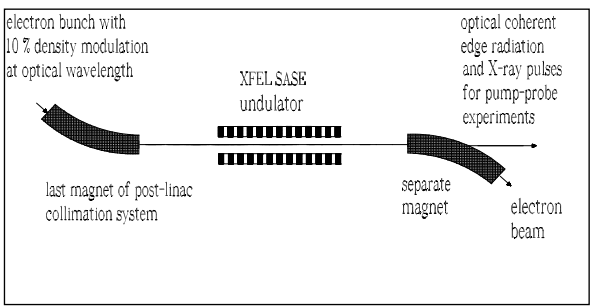

(c)

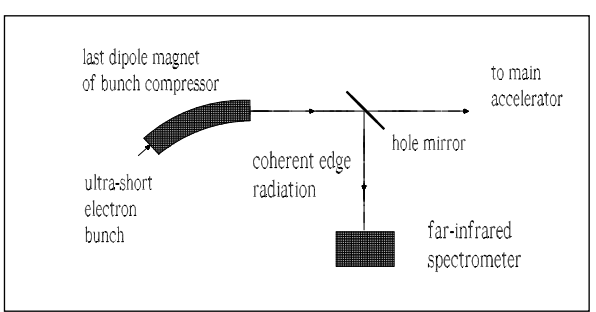

(d)

\begin{tabular}{|ll|}
\hline $\begin{array}{l}\text { ultra high gradient } \\
\text { laser plasma } \\
\text { accelerator }\end{array}$ & $\begin{array}{l}\text { ultra short } \\
\text { electron } \\
\text { bunch }\end{array}$ \\
$\begin{array}{l}\text { coherent edge } \\
\text { radiation }\end{array}$ & $\begin{array}{l}\text { optical } \\
\text { spectrometer }\end{array}$ \\
\hline
\end{tabular}

Figure 1: Four main types of edge radiation setups: (a) Far-infrared beamline for synchrotron radiation source using edge radiation. (b) Arrival-time monitor for XFEL source using optical coherent edge radiation. (c) Electron bunch length monitor for XFEL using far-infrared coherent edge radiation. (d) Ultra-short electron bunch diagnostic for laser-plasma accelerator facility using optical coherent edge radiation.

radiation wavelength $\lambda$, the radius of the bend $R$, the relativistic Lorentz factor $\gamma$, the length of the straight section $L$ and, additionally, the position of the observation plane down the beamline, $z$. We work in the far zone, operatively defined as a region where $z$ is large enough, so that the simulated spectral energy density per unit angle does not show dependence on $z$ anymore.

ER carries advantages over bending magnet radiation in the limit for $\lambda / \lambda_{c} \gg 1$, where $\lambda_{c} \sim R / \gamma^{3}$ (here $\lambda=\lambda /(2 \pi)$ is the reduced wavelength) is the critical wavelength of bending magnet radiation. We will work, therefore, in this limit. We set $\gamma=3.42 \cdot 10^{4}(17.5 \mathrm{GeV})$, $R=400 \mathrm{~m}$, which are typical values for XFELs. Note that in this case $\lambda_{c} \simeq 0.1 \AA$. Here we take $\lambda=400 \mathrm{~nm}$.

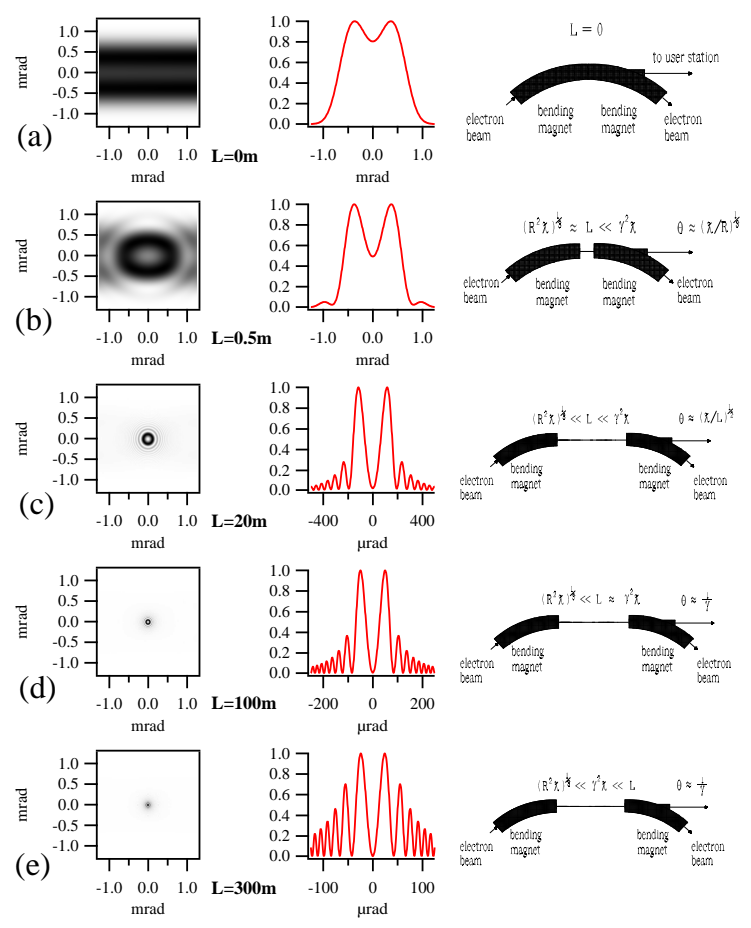

Figure 2: Calculations of the effect of bends separation on the directivity diagram of the radiation. The bending magnet radius $R=400 \mathrm{~m}$, the relativistic factor $\gamma=3.42 \cdot 10^{4}$, and the wavelength of interested $\lambda=400 \mathrm{~nm}$ are fixed, while the straight section length varies from $L=0$ up to $L \gg \gamma^{2} \lambda /(2 \pi) \simeq 100 \mathrm{~m}$. In this setup (as well as in all others in this paper) $\lambda \gg \lambda_{c} \simeq 0.1 \AA$. Case (a) is a bending magnet setup. Case (b) is a complex setup, where the radiation beam divergence is practically the same as in (a). Case (c) illustrates an ER setup. Bending magnet separation dramatically lowers the radiation beam divergence. (d) Optimal bending magnet separation. The straight section length $L \simeq \gamma^{2} \lambda /(2 \pi)$ corresponds to a radiation beam divergence $\theta \simeq 1 / \gamma$. (e) Further increase of $L$ only leads to the appearance of finer structures in the radiation profile. 2D plots on the left show the spectral energy density per unit angle as a function of the horizontal and vertical angles $\theta_{x}$ and $\theta_{y}$ for various lengths of the straight section. Middle plots are obtained cutting the 2D angular distributions at $x=0$. Right plots show a schematic of the considered layout.

We begin with the case $L=0$ (bending magnet), and we increase the straight section length (see Fig. 2). As one can see from the figure, radiation becomes more collimated, up to about $L \simeq \gamma^{2} \lambda \simeq 100 \mathrm{~m}$ (case (d)), where the collimation angle reaches $1 / \gamma \sim 30 \mu \mathrm{rad}$. Further increase of $L$ only leads to the appearance of finer structures in the radiation profile. It is important to remark that the total number of photons in the $\pm 1 \mathrm{mrad}$ window shown in Fig. 2(a) is roughly the same as in the $\pm 100 \mu \mathrm{rad}$ window in Fig. 2(d). It is clear that the length of the straight section $L$ is strongly related with the collimation of the radiation. 
To study ER further we apply similarity techniques. They allow one to reduce the number of parameters to a few dimensionless ones that are directly linked to the physics of the process, and that control it in full. Such parameters are found by analysis of the underlying equations characterizing the system under study and allow one to obtain general properties of the ER process. For the setup in Fig. 1(a), two dimensionless parameters controlling the radiation characteristics can be extracted from Maxwell's equations:

$$
\delta \equiv \frac{\sqrt[3]{R^{2} \lambda}}{L}, \quad \phi \equiv \frac{L}{\gamma^{2} \lambda} .
$$

The detector is supposed to be far away from the source so that the above-given definition of far-zone holds.

The most important general statement concerning ER is that all possible situations correspond to different values of the two dimensionless parameters $\delta$ and $\phi$.

Note that the working limit $\lambda / \lambda_{c} \gg 1$ means $\phi \cdot \delta \ll 1$ in terms of dimensionless parameters. For any two cases characterized by the same values of $\delta$ and $\phi$, the spectral energy density per unit angle from the setup in Fig. 1(a) will "look" the same in terms of angles scaled to $\sqrt{\lambda / L}$, i.e. $\hat{\theta}=\theta / \sqrt{\lambda / L}$. In other words, data for different sets of problem parameters corresponding to the same values of $\delta$ and $\phi$ reduce to a single curve when properly normalized. We tested the scaling properties of ER by running numerical simulations with the first principle computer code SRW. We used two different sets of dimensional parameters corresponding to the same case in terms of parameters $\delta$ and $\phi$, and we checked that the spectral energy density per unit angle normalized to their maximal values are identical.

When $\delta \sim 1$, the presence of the bending magnet radiation strongly influences the radiation profile. When $\delta$ decreases up to $\delta \ll 1$, one can neglect bending magnet contributions: what is left in this case is ER. These situations are realized, for example, if one works at fixed $\lambda, \gamma$ and $R$ while increasing the length $L$ as in the case of Fig. 2. It follows that $\delta$ is responsible for the relative weight of ER and bending magnet radiation contributions in the radiation profile. Since we are interested in ER emission, it is natural to consider more in detail the limit for $\delta \ll 1$. In this case, results are independent on the actual value of $\delta$, and the only parameter left is $\phi$.

In the limit for $\phi \ll 1$, the opening angle of the radiation is independent of the actual value of $\phi$ too. In this case one talks about a self-similar behavior of the profile of the spectral energy density per unit angle, which asymptotically approaches the self-similar form $I / I_{\max }=F\left(\hat{\theta}_{x}, \hat{\theta}_{y}\right)$. Note that the separation distance $L$ between the bends dramatically lowers the radiation beam divergence, but the characteristic angle of emission is still larger than $1 / \gamma$. In fact, radiation peaks at $\theta \simeq 2.2 \sqrt{\lambda / L}$. When $\phi$ increases, radiation becomes better and better collimated, up to angles $\theta \sim 1 / \gamma$. This happens for values $\phi \simeq 1$. Radiation has reached the best possible collimation angle and further increase of $\phi$ only modifies fine structures in the radiation (a)

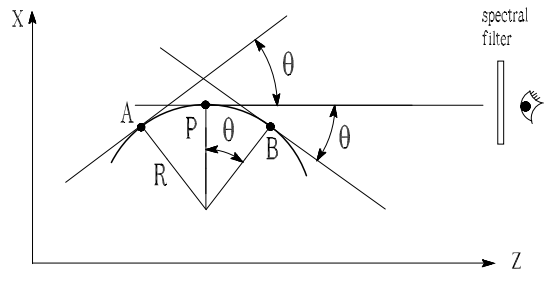

(b)

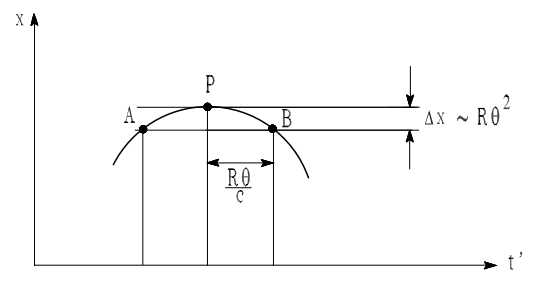

(c)

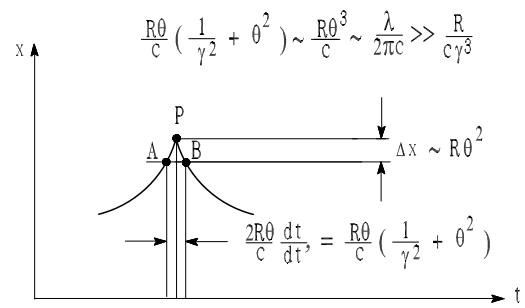

Figure 3: Geometry for SR from a bending magnet [adapted from K.-J. Kim, Characteristics of Synchrotron Radiation, in Phys. of Part. Acc. 184, AIP Conf. Proc., Am. Inst. of Phys., New York, 1984].

profile.

\section{Qualitative Description}

It is possible to present intuitive arguments to explain why all problem parameters $(R, \gamma, L$ and $\lambda)$ are effectively grouped in $\delta$ and $\phi$. Let us consider first the parameter $\delta$. By definition, $1 / \delta$ is a measure of the straight section length $L$ in units of a characteristic length $\sqrt[3]{R^{2} \lambda}$.

To explain the meaning of the quantity $\sqrt[3]{R^{2} \lambda}$, we consider Fig. 3(a), and we focus on the region of parameters $\lambda \ll R$ and $\gamma^{2} \gg 1$. A posteriori, this region of parameters will turn out to correspond to an angular dimension along the trajectory $2 \theta \ll 1$ within the bending magnet. Radiation from an electron passing through the setup is observed through a spectral filter by a fixed observer positioned on the tangent to the bend at point $P$. Electromagnetic sources propagate through the system, as a function of time, as shown in Fig. 3(b). However, electromagnetic signals emitted at time $t^{\prime}$ at a given position $x\left(t^{\prime}\right)$ arrive at the observer position at a different time $t$, due to the finite speed of light. As a result, the observer in Fig. 3(a) sees the electromagnetic source motion as a function of $t$. What one needs to know, in order to calculate the electric field, is the apparent motion $x(t)$ shown in Fig. 3(c), which is a hypocycloid, and not the real motion $x\left(t^{\prime}\right)$. In fact, the electric field at the observa- 
tion point is proportional to the second derivative of the $\mathrm{x}$ coordinate with respect to the retarded time $t$, because the observer sees everything as delayed. We discuss the case when the source is heading towards the observer. Using the fact that $\theta \ll 1$, one obtains the well-known relation $d t / d t^{\prime}=1 / 2 \cdot\left(1 / \gamma^{2}+\theta^{2}\right)$. The observer sees a timecompressed motion of the sources, which go from point $A$ to point $B$ in an apparent time corresponding to an apparent distance $2 R \theta d t /\left(d t^{\prime}\right)$. Let us assume $\theta^{2}>1 / \gamma^{2}$. In this case one has $2 R \theta d t /\left(d t^{\prime}\right) \simeq R \theta^{3}$. Obviously one can distinguish between radiation emitted at point $A$ and radiation emitted at point $B$ only when $R \theta^{3} \gg \lambda$, i.e. for $\theta \gg(\lambda / R)^{1 / 3}$. This means that, as concerns the radiative process, we cannot distinguish between point $A$ and $B$ on the bend such that $R \theta \lesssim\left(R^{2} \lambda\right)^{1 / 3}$. It does not make sense at all to talk about the position where electromagnetic signals are emitted within $L_{f b}=\left(R^{2} \lambda\right)^{1 / 3}$ (here we are assuming that the bend is longer than $\left.L_{f b}\right)$. This characteristic length is called the formation length for the bend. The formation length can also be considered as a longitudinal size of a single-electron source. Note that a single electron always produces diffraction-limited radiation $d \cdot \Delta \theta \sim \lambda$, $d$ being the transverse size and $\Delta \theta$ the divergence of the source. Since $d \sim L_{f b} \Delta \theta$, it follows that the divergence angle $\Delta \theta$ is strictly related to $L_{f b}$ and $\lambda: \theta \sim \sqrt{\lambda / L_{f b}}$. One may check that, using $L_{f b} \sim \sqrt[3]{R^{2} \lambda_{c}}$, one obtains $\theta \sim \sqrt[3]{\lambda / R}$; in particular, at $\lambda \sim \lambda_{c} \sim R / \gamma^{3}$ one obtains $\theta \sim 1 / \gamma$, as is well-known for bending magnet radiation. Let us now consider the case of a straight section of length $L$ inserted between the two halves of a bend. Since we cannot distinguish between points within $L_{f b}$, the case $L=0$ is obviously indistinguishable from the case $L \ll L_{f b}$. Significant deviations from the bending magnet case are to be expected when $L \gtrsim L_{f b}$, i.e. when $\delta \lesssim 1$. This hints to the fact that $\delta$ is responsible for the relative weight of ER and bending magnet radiation contributions in the radiation profile.

Let us now discuss the parameter $\phi$. By definition, $\phi$ is a measure of the straight section length $L$ in units of a characteristic length $\gamma^{2} \lambda$. One can still use the same reasoning considered for the bend to define a region of the trajectory where it does not make sense to distinguish between different points. In the case of a straight section of length $L$ connecting $A$ and $B, d t / d t^{\prime}=1 /\left(2 \gamma^{2}\right)$. It follows that the apparent distance $A B$ is equal to $L /\left(2 \gamma^{2}\right)$. Since it does not make sense to distinguish between points within the apparent electron trajectory such that $L /\left(2 \gamma^{2}\right) \lesssim \lambda$, one obtains a critical length of interest $\sim \gamma^{2} \lambda$. This hints to the fact that for values $\phi \simeq 1$ radiation has reached the best collimation angle.

Note that for ultrarelativistic systems in general, the formation length is always much longer than the radiation wavelength. This is related with a large compression factor $d t / d t^{\prime}$. For comparison, in the case of non-relativistic motion the compression factor $d t / d t^{\prime} \simeq 1$, and the formation length is simply of order of the radiation wavelength. The counterintuitive result follows, that for ultrarelativistic systems one cannot localize sources of radiation within a macroscopic part of the trajectory. Following this intuitive introduction, in [1] we gave a rigorous derivation of the ER properties, with their analytical asymptotes and their applicability region both in terms of a Fourier optics, namely based on the picture of a single laser-like beam in the middle of the undulator, or two laser-like beams at the edges of the straight section.

\section{APPLICATIONS}

Based on this fundamental case study one can analyze a more complicated setup, consisting of an undulator preceded and followed by two straight sections and two bends (see Fig. 1(b)). ER from this kind of setup is commonly known as Transition Undulator Radiation (TUR). The first study on TUR appeared more than a decade ago, when it was pointed out for the first time that, since an electron entering or leaving an undulator experiences a sudden change in longitudinal velocity, highly collimated radiation with broadband spectrum, similar to transition radiation, had to be expected in the low-frequency region in addition to the usual UR. More recently, TUR has been given consideration in the framework of X-ray Free-Electron Laser (XFEL) projects. For example, an arrival-time monitor for XFELs using infrared coherent ER from a setup similar to that in Fig. 1(b) has been proposed, which should be used for pump-probe experiments with femtosecond-scale resolution. In view of these applications, there was a need to extend the characterization of TUR to the near-zone, and to the coherent case. From this viewpoint, specification of what precedes and follows the undulator is of fundamental importance. As has been recognized for TUR many years ago, if this information is not known, any discussion about the intensity distribution of TUR is meaningless. According to our approach, the two straight sections and the undulator in the setup in Fig. 1(b) will be associated to virtual sources with plane wavefronts. The field from the setup can then be described, in the near as well as in the far-zone, as a superposition of laser-like beams, radiating at the same wavelength and separated by different phase shifts. Detailed results are shown in [1].

\section{REFERENCES}

[1] G. Geloni, V. Kocharyan, E. Saldin, E. Schneidmiller and M. Yurkov, Nuclear Instruments and Methods in Physics Research A 605 (2009) 409429. 